\title{
Application of Virtual Reality Technology in Physics Experiment Teaching
}

\author{
Huazhong Wang \\ College of Science, Yunnan Agricultural University, Kunming, Yunnan Province, 650225, China \\ whzhong8861@.sina.com
}

Keywords: Virtual reality; physics teaching; application research.

\begin{abstract}
To explore the application of virtual experiment in physics teaching, the virtual reality constructivism, hybrid learning theory, interaction theory and the used techniques were analyzed. Then, the whole process of virtual experiment system from the design to the realization was explained in detail, and the representative physical experiment was selected as the object of the virtual experiment application research. The application of virtual reality in the middle school physics teaching was analyzed. The research results showed that the virtual reality in experimental teaching was beneficial to improve teachers' teaching quality and enhance students' autonomous learning ability. It is concluded that the virtual experiment has prominent teaching significance in physics teaching.
\end{abstract}

\section{Introduction}

With the rapid development of computer technology and modern education technology, the application of virtual reality technology has become more popular. As the third scientific research method following theoretical research and experimental research, the virtual reality plays an increasingly important role in social development and scientific and technological progress. At the same time, it represents the development direction of scientific research methods [1]. Continuously deepening reforms in education and teaching put forward higher requirements for experimental teaching in cultivating students' scientific research capabilities and overall quality. The teaching content, teaching mode, and teaching method of experimental teaching are subject to innovation and improvement [2]. The mature development of virtual reality technology and the urgent need of experimental teaching reform make the application of virtual experiment in teaching go from theoretical research to practical application.

In this paper, the development status of virtual reality technology both at home and abroad is introduced, and the theoretical foundations of virtual reality constructivism, hybrid learning theory and interaction theory, as well as the used technologies are analysed. In the end, the application research of virtual reality in junior middle school physics teaching is summarized and the direction of further research is put forward.

\section{Literature Review}

Virtual reality technology originates in the United States, and the research level in the United States can represent the development level of virtual reality technology [3]. Initially, the application of virtual reality technology in the United States mainly focuses on the military. With the development of virtual reality technology and the reduction of US military spending, these technologies have gradually become civilian. In the 1980s, the achievements of National Aeronautics and Space Administration (NASA) and the United States Department of Defense (USDOD) in the research of virtual reality technology attracted people's attention to virtual reality technology [4]. In 1984, Dr. J. Humphries and M. McGreevy of the virtual planet exploration laboratory at NASA Ames Research Center organized the visual display of virtual environment and used for the detection of Mars in the United States. A Martian three-dimensional virtual model was constructed using information from the Mars ground returned by the Mars probe. 
Foreign researches on virtual reality technology started earlier. Their theories are relatively mature and they have made great breakthroughs in practical applications. They have been involved in various fields such as military, space technology, medicine and even entertainment and achieved certain achievements. Many universities and companies in China are also conducting research on virtual reality technology. Beijing University of Aeronautics and Astronautics is one of the earliest organizations to study this technology in China. They established the virtual reality technology and system and state key laboratory in 2007. Finally, a lot of application systems such as the simulation training system and the creative realistic environment of opening ceremony of the Beijing Olympic Games were developed [5]. Harbin Institute of Technology solves the synthesis of facial expressions and lip synthesis in human behaviours. It also can synthesize the face images. The current research is focusing on the synchronization of human speech and intonation. The CAD \& CG lab at Zhejiang University has applied virtual reality technology in the simulation and real-time roaming of the building environment [6]. Other universities such as Tsinghua University and China University of Science and Technology are also actively involved in this research field and have achieved some research results.

\section{Methodology}

\subsection{Virtual Experiment Design Steps}

The virtual reality technology is used as a means to complete the design of the virtual experiment system. First, we must plan carefully in advance. This plan should be designed in detail, and it should be conducted step by step. The specific design steps are shown in figure 1 .

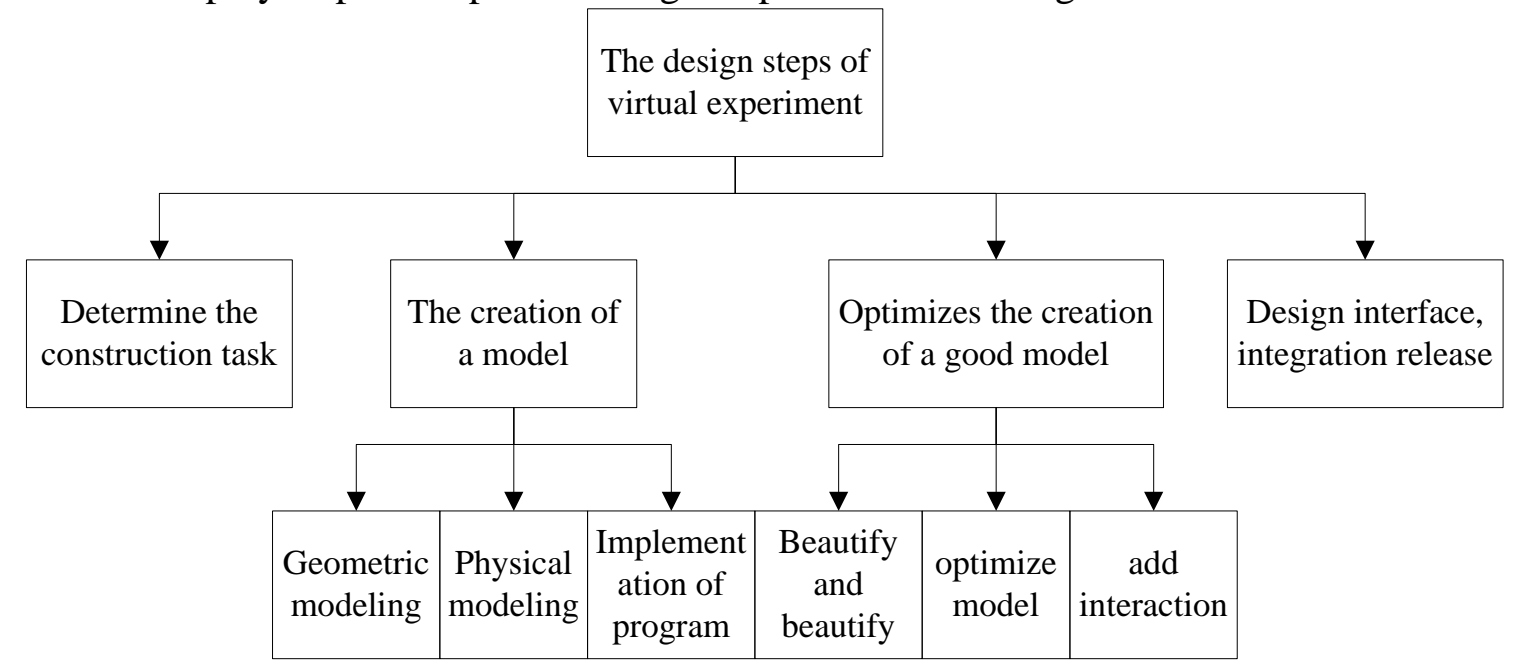

\subsection{Virtual Experiment Design Principle}

Figure 1. The design steps of virtual experiment

The interconnectedness of the various components within the system: If one of the elements changes, the other elements will change accordingly. Only mutually related elements can form a whole. For a teaching system, any two of the teaching elements are also related, and a series of related elements constitute a teaching whole. The system function is not a simple addition of the functions of each element. The integrity of the system reflects that it also has some new functions. These functions cannot be embodied when the elements of the system are isolated from each other.

The experimental design is the careful planning of the entire program and its specific links, the overall structure and related aspects of the system. A virtual system [7] is a collection of structures and functions organized by various elements in a certain way to achieve a specific goal. Therefore, the design itself requires integrity and system.

The elements that make up the virtual experiment system are different and the system is complex. To simulate a system, the system cannot simulate running if any of these are ignored. However, the virtual experiment system is also open, and we can continue to supplement and perfect it. 
The so-called scientific nature requires the correct application of educational ideology and educational principles, which not only conforms to the intrinsic connection and regularity of education and teaching, but also reflects the intrinsic connection and regularity of subject knowledge. The two are in harmony and complement each other. The design process is required to be straightforward, clear, and rationality and rigor. The so-called artistry requires that the teaching design is refined, perfect, effective, and original, which can greatly stimulate students' learning emotions, satisfy their learning desires, and create a harmonious and pleasant atmosphere between teachers and students.

\subsection{Virtual Experiment Structure}

The structure of virtual experiment includes three parts: virtual experiment human-machine interface, experiment processing system and integrated management system.

The virtual experiment man-machine interface [8] is the interface for input and output, and it is the window for man-machine interaction. The user interacts with the system through a virtual experiment human-machine interface. After the human-machine interface analyses and recognizes the user's input information, it is converted into commands and passed to the experimental processing system. Human-machine interface is generally divided into three parts: dialogue environment, system management and data entity. The dialogue environment provides interactive mode. The system management is responsible for user registration and usage record. The data entity provides support for dialogue environment and system management. It is composed of user information database, interactive rules library and other components. When the system only needs the query operation, the man-machine interface can directly call the database and the knowledge base without going through the problem solving and the integrated management system.

The experimental processing system is mainly composed of three parts: the model construction part, the data processing part and the task execution part. The integrated management system is the management control part of the virtual experiment. According to the commands of the experiment processing system, it calls the resources of each library. At the same time, it is responsible for the protection of data resources. It not only connects and coordinates the work of various parts of the system, but also realizes the process between various modules.

\section{Results and Discussion}

\subsection{Virtual Experiment in Experimental Teaching Mode}

As shown in figure 2, the virtual experiment is used to prepare for the actual experiment. The teacher asks the learner to define the experimental principle, the purpose and the requirements through virtual experiments, and then follow the requirements and perform the thinking and answering. After being familiar with virtual experiments, learners can effectively avoid mis-operations and prevent instrument damage when they do real experiments.

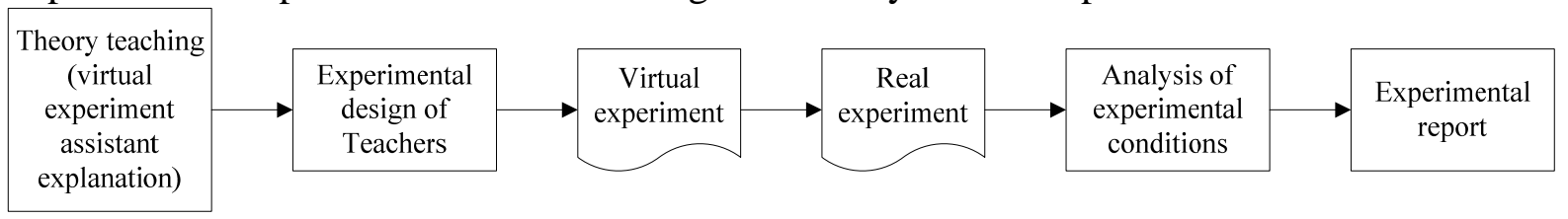

Figure 2. Steps to do virtual experiments before finishing real experiments

As shown in figure 3, a virtual experiment is performed after completing the real experiment. In middle school physics experiment teaching, teachers can lead students to do real experiments first, and then do virtual experiments. This method mainly plays a role in reviewing experiments and consolidating memory. This method can effectively help students understand the experimental principles, digest the experimental content and analyse the problems in the experiment. Students are more impressed with some difficult experiments. The problems in the process of doing real experiments need to be considered when doing virtual experiments. 


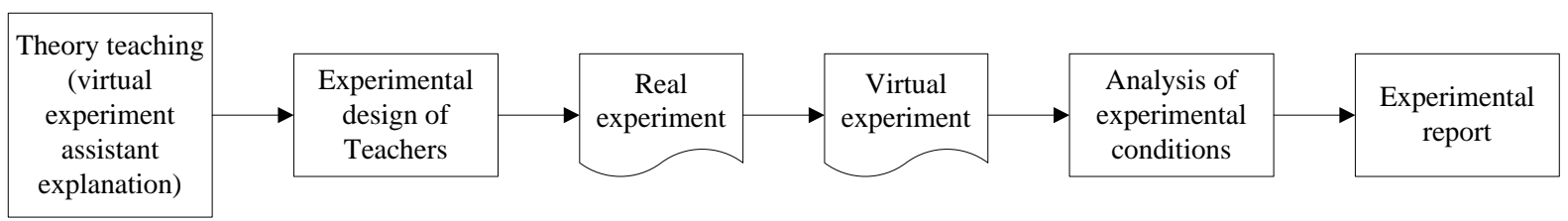

Figure 3. Steps to do virtual experiments after finishing a real experiment

Figure 4 is the virtual experiment process. For some secondary school, the experimental conditions are limited and cannot open a real physics experiment. In high school physics experiment teaching, there are some large investment. There is a certain risk of the experiment. The real experiments can replace the virtual experiments. This model can make up for the inability to open a real experiment and satisfy the learner's desire for learning to a certain extent. This model is based on the traditional experimental teaching model. It inherits the advantages of the traditional experimental teaching model, further gives full play to the advantages of virtual technology in experimental teaching. Finally, it fully cultivates students' experimental and practical abilities.

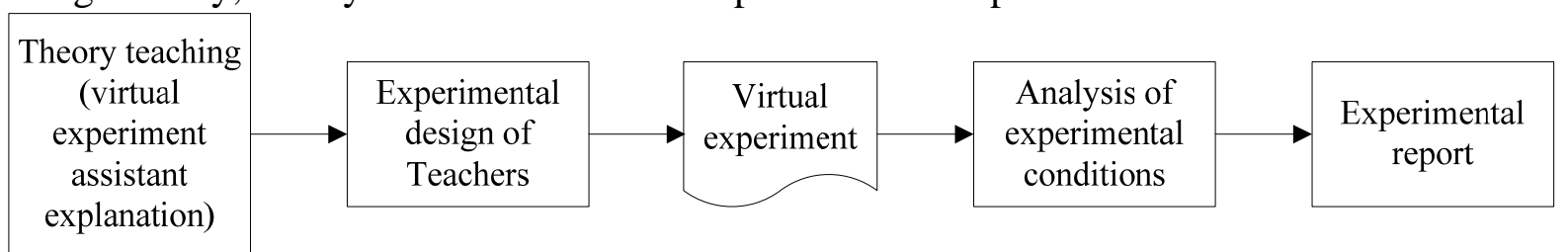

Figure 4. Steps to do virtual experiments

\subsection{Application of Virtual Experiment in Physics Experiment Teaching}

To understand the application effect of virtual experiment in physics experiment teaching, this experiment combines the questionnaires, comparative study and on-site observations. Two junior high school classes are selected as test samples. One class adopts the virtual experiment mode of teaching, the other uses the traditional mode of teaching. Through the evaluation of students' physics learning interest and problem exploration consciousness and other indicators, the application of virtual experiments in the physics teacher experimental teaching is evaluated and reflected.

To further ensure the validity of the questionnaire, the expert survey method is used to formulate an expert survey questionnaire. The expert questionnaire uses five evaluation forms, including high, very high, general, low, and very low.

$$
\begin{gathered}
\rho_{x y}=r(x, y)=0.905 \\
\text { P value }<0.01
\end{gathered}
$$

In this study, the back test is used to test the credibility of the questionnaire. The investigators are re-investigated during the process of questionnaire issuance. The formula 1 and formula 2 showed that the questionnaires were highly relevant. The questionnaire is counted and analysed. Then, the following results are obtained:

As shown in figure 5, two questions are analysed. One is "Do you like physical class?" The other is "Do you like physical experiment?" The result shows that the introduction of virtual experiments in junior high school physics teaching can improve students' interest in learning. 


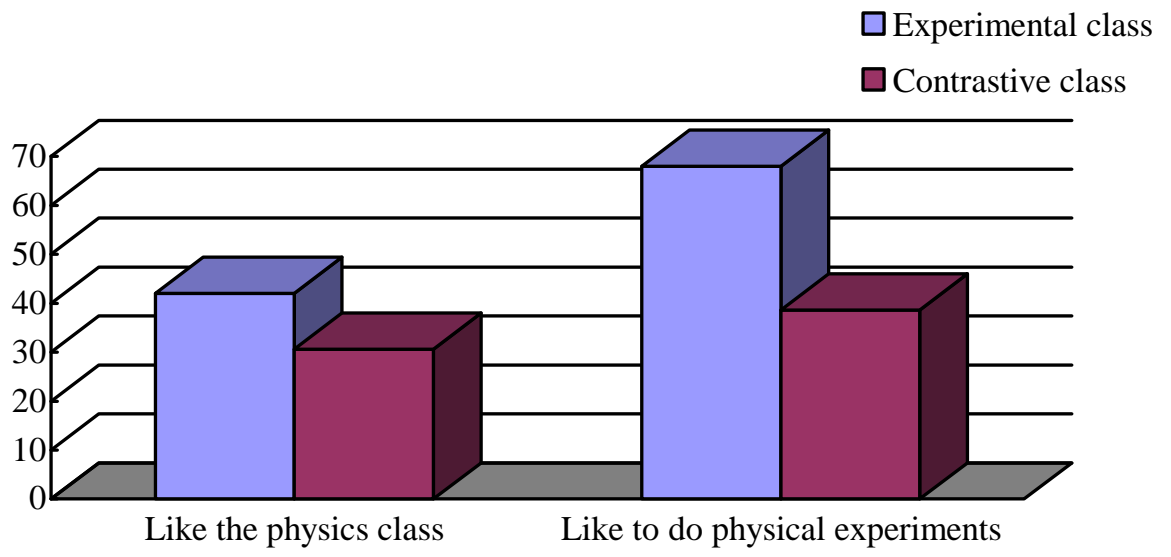

Figure 5. Comparison of interest in physical learning (the proportion of class numbers)

As shown in figure 6, two questions are analysed. One is "Are you interested in self-exploration group? The other is "What would you do when you facing a problem?” Finally, the students' awareness of innovation and collaboration is analysed. The result shows that students in the experimental class have stronger collaboration and innovation than contrast classes.

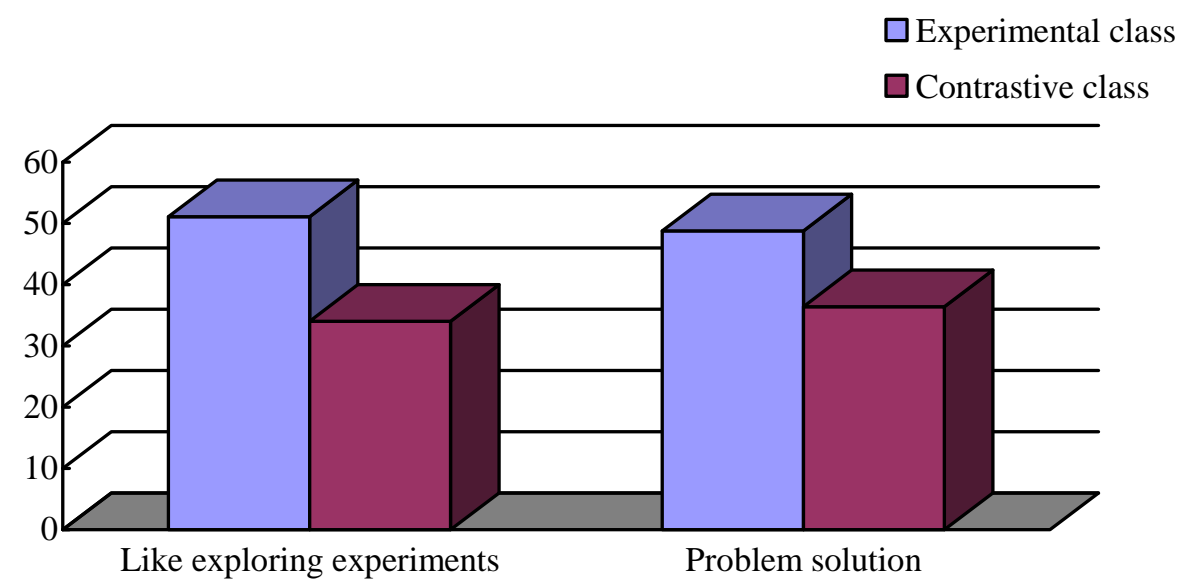

Figure 6. Comparison of question consciousness

\section{Conclusion}

Based on a large number of literature research, the necessity and feasibility of using virtual experiments to solve these problems are put forward. The research status of virtual reality technology at home and abroad is summarized and analysed. The support theory of a large number of virtual experimental systems is studied. The technical description of virtual experiments is mainly based on the determination of system design goals and system construction ideas. Meanwhile, the system design and content design of the virtual experimental system are designed in detail. In addition, the research on the instructional design of teaching aids for virtual experiment assistants was also achieved. The advantages and disadvantages of virtual experiments in physics teaching are summarized. At the same time, the issues needing attention in the application were also summarized.

\section{References}

[1]. Fang M, Yang B, Wang R, et al. The application of virtual reality technology in the experimental teaching of acute critical care. Chinese nursing education, 2015, 2(11), pp. 145-150.

[2]. Zhang L, Wang B. Development of experimental platform for electrical engineering based on virtual reality technology. Experimental technology and management, 2015, 32 (2), pp. 110-111. 
[3]. Zhang C, Yang T, Wang Q, et al. Design and implementation of the passive three-dimensional virtual reality experimental teaching system. Microcomputer application, 2015, 31 (9), pp. 279-292.

[4]. Xiang Y, Fan Y, Wang F, et al. The role of virtual laboratory in psychological experiment teaching. Experimental technology and management, 2015, 32 (12), pp. 52-55.

[5]. Fu W. The exploration of the rural sports teaching mode of "body" and "heart". Modern reading, 2013, 1(3), pp. 6-7.

[6]. Guo R. A new model of physical education and the cultivation of quality education. Modern reading, 2013, 1(3), pp. 13-18.

[7]. Sang Y., Zhu Y., Zhao H., Tang M. Study on an interactive truck crane simulation platform based on virtual reality technology. International Journal of Distance Education Technologies, 2016, 14(2), pp. 64-78.

[8]. Koeva M., Luleva M., Maldjanski P. Integrating spherical panoramas and maps for visualization of cultural heritage objects using virtual reality technology. Sensors, 2017, 17(4), pp. 829. 